\title{
COMMENTARY
}

\section{Randomized-Control Screening Trials to Lower Gall Bladder Cancer Mortality in High Risk Populations}

\author{
Manigreeva Krishnatreya ${ }^{1 *}$, Amal Chandra Kataki²
}

\begin{abstract}
Gall bladder cancer is generally fatal. The high morbidity and mortality due to gall bladder cancer exerts a significant impact on efforts towards cancer control in high risk populations of the World and a rationale program for control of gall bladder cancer mortality has remained as an unmet need in these populations. Currently there are no effective strategies for controlling gall bladder cancer mortality. This mini review is to highlight the need and feasibility for secondary prevention of gall bladder cancer by screening in high risk populations. A way forward is to assess the role of secondary prevention of gall bladder cancers by conducting randomizedcontrolled screening trials in high risk populations.
\end{abstract}

Keywords: Gall bladder cancer - mortality - screening trial - high risk population

Asian Pac J Cancer Prev, 17 (4), 2325-2327

\section{Introduction}

Gall bladder cancer (GBC) is the most common malignancy that arises from the biliary tract. The incidence of GBC is particularly highin some regions of the world. GBC is common in the Indian subcontinent (India, Pakistan), South America (Chile, Bolivia, and Columbia), East Asia (Korea, Japan) and central Europe (Slovakia, Poland, and Czech Republic) and it is relatively uncommon in the rest of the world (Randi et al., 2006; Randi et al., 2009). The incidence rates of gallbladder cancer have reached epidemic levels in high risk regions and ethnicities. The global variation in the incidences of gall bladder cancers can be attributed to genetic, racial and environmental factors.

The fatality of GBC is very high, because almost all the cases are diagnosed in advanced stages with exception of a few cases which are diagnosed incidentally after cholecystectomy for gall stone disease. Early-stage tumors are often curable with a proper resection (Jayaraman and Jarnagin, 2010), and cholecystectomy remains the only option for cure. The frequency of cholecystectomy for gallbladder disease is a factor for reduction of mortality due to GBC (Wistuba and Gazdar, 2004). The survival in GBC is abysmally poor with an overall mean survival of 6 months (Lai and Lau, 2008) and overall 5-year survival of less than 5\% (Duffy et al., 2008). But, there was a significant improvement in the median survival to almost 16 months in incidental cases of GBC (Duffy et al., 2008). Also, patients with incidental GBC showed better prognosis (Cha and Bae, 2014).

The incidence of incidental GBCs is around $0.25 \%$ in low risk regions of the world (Emmett et al., 2015), but in region with high risk population, detection rate of incidental GBC was as high as $3 \%$ (Waghmare and Kamat, 2014). There is a study which advocated the role of prophylactic cholecystectomy in females with asymptomatic gall stones for lowering GBC mortality in high risk population (Mohandas and Patil, 2006). Gall stone disease has been implicated as a major risk factor in the development of GBC in high risk population (Dwivedi et al., 2015). For now, the main focus has been to improve the survival in patients with gall bladder cancer by exploring combination chemotherapy as adjuvant therapies, like gemcitabine and cisplatin combination chemotherapy.

Despite advances in surgery and adjuvant treatment for $\mathrm{GBC}$, it is also imperative to focus on primary prevention or secondary prevention by screening. Recently, markers to predict the risk of GBC in patients with gall stone diseases have been suggested for screening (Mishra et al., 2015). This commentary is to highlight the need for secondary prevention of GBC by screening in high risk populations, and to discuss whether such screening trials are a feasible option.

\section{Screening Feasibility}

GBC has a long natural history from metaplasia progressing to dysplasia, carcinoma in situ, and then, invasive cancer (Lazcano-Ponce et al., 2001). The natural history of a disease is one of the important determinants for carrying out a screening test for its early detection by screening. Thus, GBC should be viewed from the 


\begin{tabular}{crc}
\hline Serial number & Criteria & Check \\
\hline 1 & The condition should be an important public health problem & Yes \\
2 & There should be accepted treatment for patients with recognized disease & Yes \\
3 & Facilities for diagnosis and treatment should be available & Yes \\
4 & There should be a latent or early symptomatic stage & Yes \\
5 & There should be a suitable test or examination & Yes \\
6 & The test should be acceptable to the population & Yes \\
7 & The natural history of the condition from latent to declared disease should be understood & Yes \\
8 & There should be an agreed policy on who to treat as patients & Yes \\
9 & The cost of case finding should be economically balanced & Yes \\
10 & Case finding should be a continuous, and not 'once and for all" project & Yes \\
\hline
\end{tabular}

perspective of a screening test for its early detection. Ultrasonography (USG) should be the first step in the early diagnosis of gall bladder cancers radiologicaly in an epidemiologic setting (incidental finding) (RodriguezFernandez et al., 2006). Ultrasonography is a subjective investigation based on expertise, quality of the machine, the patient's obesity as well as the fasting state of the gall bladder. Although, USG has a high sensitivity for the detection of tumor, it is limited in the diagnosis of early lesions and is unreliable for staging. To overcome the subjective assessment of USG, it is advisable to followup the USG diagnosis with a computed tomogram (CT) scan or PET scan to augment the findings. Radiological presentation can be mass occupying or replacing the lumen, focal or diffuse asymmetric thickening and/ or intra-luminal polyp. Radiologists should closely scrutinize the gallbladder, particularly in patients who are at increased risk of developing gallbladder carcinoma, for subtle morphologic abnormalities that may indicate malignancy (Furlan et al., 2008). And, the final diagnosis of incidental GBC has to be made by histological examination after a cholecystectomy. A major concern of a screening trial is both high numbers of false positive and false negative findings.

\section{Conclusions}

Currently there is no randomized-controlled screening trial that has been conducted to see the role of screening in lowering GBC mortality. If the cases of incidental GBC can be identified by a screening radiological suspicion followed by its surgical removal (cholecystectomy), the mortality due to it can be reduced. Of all incidental GBCs, $30 \%$ of patients had pre-operative subtle radiological evidences like wall thickening (Rammohan et al., 2014), and such radiological evidences should provide the leads in high risk populations. Also, asymptomatic detection of gall stones should warrant a cholecystectomy to abrogate the risk of GBC (Dutta, 2012). The debate is there as to the role of prophylactic cholecystectomy in asymptomatic gall stone disease. One of the arguments against screening trial for GBC would be unnecessary cholecystectomies due to the overlap of cholecystitis and gall bladder polyps with GBC in a screening trial. The concern with cholecystectomy is its attendant morbidity and mortality, as any pre-operative suspicion of $\mathrm{GBC}$ would require an open cholecystectomy and laparoscopic surgery is relatively contraindicated. A simple cholecystectomy is reserved for only T1a disease and it offers a surgical (Reid et al., 2007), however this may not be the case for all incidental cases of GBC. Laparoscopic surgery is much safer with a negligible morbidity in comparison. However despite such worries, symptomatic cholecystitis and asymptomatic gall bladder polyps would necessitate a cholecystectomy or such patients are better advised for cholecystectomy especially in settings when close serial follow-up may not be feasible in high risk populations (Andren-Sanberg, 2012). An issue that surrounds any screening trial is methodological pitfall in determining the benefits of trials. It is unjust to implement a screening program that is costly to the community and to the individual, when its benefits are not certain in the population (Juffs and Tannock, 2002). Still randomized-control trials for cancers to determine the effects of screening on cancer-related mortality are done. Another issue will be that a large number of populations will have to be screened in order to identify few cases of GBC. In a mass population-based screening of general population in Japan the detection rate was only $0.011 \%$ and most of whom had advanced GBC (Inui et al., 2011). So, a randomized-control screening trial to assess the impact of screening in lowering GBC disease-specific mortality should be done before implementing a population-based strategy of mass screening.

On evaluation of screening and GBC with the basic criteria for screening of the World Health Organization (Wilson and Jungner, 1968), the ten point checklist results were favorable for starting a screening trial as shown in table 1 . The high morbidity and mortality due to gall bladder cancer is having a significant impact in the efforts towards cancer control in high risk regions of South America and Asia, and a rationale program for control of GBC mortality has remained as an unmet need in these populations. Secondary prevention of GBC by cholecystectomy in endemic areas and in patients at risk should be clarified, with its inherent risks and cost (Hundal and Shaffer, 2014). A study from South America in Chilean women has shown prophylactic laparoscopic cholecystectomy could benefit the population at a low incremental cost (Puschel et al., 2002). This type of costbenefit analysis study can also be incorporated along with randomized-controlled screening trials. Furthermore, public health programs to sensitize the lay public about 
screening strategies for GBC have also been suggested (Dutta, 2012). Currently there is absence of an effective strategy to tackle this fatal cancer, there is an increasing burden of GBC in high risk regions and there is a high disease-specific mortality. Furthermore, there are limited treatment modalities currently available for better outcome results in GBC. Thus there appear four good reasons to start randomized-control screening trials to see the efficacy of screening in lowering mortality due to GBC. The randomized-controlled screening trial should be conducted in hospitals or cancer treatment centers where the facilities with the necessary expertise and safe surgery are available. The stumbling block for such a screening trial would be constraints of resources in these low-middle income countries, where this dreaded disease mostly affects its population. But, resource constraints should not be a barrier for improvement of healthcare in a society.

\section{Acknowledgements}

Authors would like to thank Cancer Research Foundation, India for providing the necessary support

\section{References}

Andren-Sanberg A (2012). Diagnosis and management of gallbladder polyps. $N$ Am J Med Sci, 4, 203-11

Cha BH, Bae JM (2014). Comparison of clinical outcomes of incidental and non-incidental gallbladder cancers: a singlecenter cross- sectional study. Asian Pac J Cancer Prev, 15 , 1281-3.

Duffy A, Capanu M, Abou-Alfa GK, et al (2008). Gallbladder cancer (GBC): 10-year experience at memorial sloankettering cancer centre (MSKCC). J Surg Oncol, 98,485-9.

Dutta U (2012). Gallbladder cancer: can newer insights improve the outcome? J Gastroenterol Hepatol, 27, 642-53

Dwivedi S, Agrawal S, Singh S, et al (2015). Association of cytochrome-17 (mspa1) gene polymorphism with risk of gall bladder stones and cancer in North India. Asian Pac J Cancer Prev, 16, 5557-63.

Emmett CD, Barrett P, Gilliam AD, et al (2015). Routine versus selective histological examination after cholecystectomy to exclude incidental gallbladder carcinoma. Ann R Coll Surg Engl, 97, 526-9.

Furlan A, Ferris JV, Hosseinzadeh K, et al (2008). Gallbladder carcinoma update: multimodality imaging evaluation, staging, and treatment options. AJR Am J Roentgenol, 191, 1440-7.

Hundal R, Shaffer EA (2014). Gallbladder cancer: epidemiology and outcome. Clin Epidemiol, 6, 99-109.

Inui K, Yoshino J, Miyoshi H (2011). Diagnosis of gallbladder tumors. Intern Med, 50, 1133-6.

Jayaraman S, Jarnagin WR (2010). Management of gallbladder cancer. Gastroenterol Clin North Am, 39, 331-42.

Juffs HG, Tannock IF (2002). Screening trials are even more difficult than we thought they were. $J$ Natl Cancer Inst, 94, 156-7.

Lai CH, Lau WY (2008). Gallbladder cancer- a comprehensive review. Surgeon, 6, 101-10.

Lazcano-Ponce EC, Miquel JF, Munoz N, et al (2001). Epidemiology and molecular pathology of gallbladder cancer. CA Cancer J Clin, 51, 349-64.

Mishra K, Behari A, Kapoor VK, et al (2015). Platelet derived growth factor-b and human epidermal growth factor receptor-2 polymorphisms in gall bladder cancer. Asian Pac
J Cancer Prev, 16, 5647-54.

Mohandas KM, Patil PS (2006). Cholecystectomy for asymptomatic gallstones can reduce gall bladder cancer mortality in northern Indian women. Indian J Gastroenterol, 25, 147-51.

Puschel K, Sullivan S, Montero J, et al (2002). Cost-effectiveness analysis of a preventive program for gallbladder disease in Chile. Rev Med Chil, 130, 447-59

Rammohan A, Cherukuri SD, Sathyanesan J, et al (2014). Incidental gall bladder cancers: are they truly incidental? World J Gastrointest Oncol, 6, 441-3

Randi G, Franceschi S, La Vecchia C (2006). Gallbladder cancer worldwide: geographical distribution and risk factors. Int $J$ Cancer, 118, 1591-602

Randi G, Malvezzi M, Levi F, et al (2009). Epidemiology of biliary tract cancers: an update. Ann Oncol, 20, 146-59

Reid KM, Ramos-De la Medina A, Donohue JH (2007). Diagnosis and surgical management of gallbladder cancer: a r eview. J Gastrointest Surg, 11, 671-81.

Rodriguez-Fernandez A, Gqmez-Rio M, Medina-Benitez A, et al (2006). Application of modern imaging methods in diagnosis of gallbladder cancer. J Surg Oncol, 93, 650-64

Waghmare RS, Kamat RN (2014). Incidental gall bladder carcinoma in patients undergoing cholecystectomy: a report of 7 cases. J Assoc Physicians India, 62, 793-6.

Wilson JMG, Jungner G (1968). Principles and practice of screening for disease. World Health Organization Public Health Papers, 34.

Wistuba II, Gazdar AF (2004). Gallbladder cancer: lessons from a rare tumour. Nat Rev Cancer, 4, 695-706. 